\title{
An Analytic and Combinatorial-Geometric Proof of a Knopp-Type Identity for Multiple Dedekind Sums
}

\author{
Kazuhito Kozuka \\ Department of Mathematics, National Institute of Technology, Miyakonojo College, Miyakonojo Miyazaki, 885-8567, Japan
}

Received: June 26, 2015 / Accepted: July 23, 2015 / Published: October 25, 2015.

\begin{abstract}
In this paper, we define multiple Dedekind sums by products of Bernoulli functions. From the Fourie expansions of Bernoulli functions, we express the Dedekind sums as series representatios. Then by a combinatorial-geometric method, we give a new proof of a Knopp-type identity for the Dedekind sums.
\end{abstract}

Keywords: Dedekind sums, Knopp's formula, combinatorial

\section{Introduction}

For $h \in \mathbf{Z}$ and $k \in \mathbf{N}$, the classical Dedekind sum $s(h, k)$ is defined by

$$
s(h, k)=\sum_{\alpha \bmod k}\left(\left(\frac{\alpha}{k}\right)\right)\left(\left(\frac{\alpha h}{k}\right)\right),
$$

where

$$
((x))=\left\{\begin{array}{l}
x-[x]-\frac{1}{2} \quad \text { if } \mathrm{x} \notin \mathbf{Z} \\
0 \quad \text { if } \mathrm{x} \in \mathbf{Z}
\end{array}\right.
$$

and the following formulas are well known:

(I) Reciprocity formula (Dedekind [14])

$$
12 h k\{s(h, k)+s(k, h)\}=h^{2}-3 h k+k^{2}+1
$$

for $h, k \in \mathbf{N}$ with $\operatorname{gcd}\{h, k\}=1$.

(II) Knopp's formula (Knopp [16])

$$
\sum_{\substack{a d=N \\ d>0}} \sum_{b=0}^{d-1} s(a h+k b, d k)=\sigma(N) s(h, k)
$$

for $N \in \mathbf{N}$, where $\sigma(N)=\sum_{\delta \mid N} \delta$.

Generalizations of Dedekind sums and formulas (2) and (3) have been studied by many mathematicians

Corresponding author: Department of Mathematics, National Institute of Technology, Miyakonojo College, Miyakonojo Miyazaki, 885-8567, Japan. E-mail: k31k@cc.miyakonojo-nct.ac.jp. extensively with many methods. For $p \in \mathbf{Z}_{\geq 0}$, let $B_{p}(X)$ be the $p$ th Bernoulli polynomial and $\bar{B}_{p}(x)$ the $p$ th Bernoulli function defined by

$$
\bar{B}_{p}(x)= \begin{cases}B_{p}(x-[x]) & \text { if } p \neq 1 \\ ((x)) & \text { if } p=1 .\end{cases}
$$

For $p, q \in \mathbf{Z}_{\geq 0}$, the sum (1) is naturally generalized as

$$
s_{p, q}(h, k)=\sum_{\alpha \bmod k} \bar{B}_{p}\left(\frac{\alpha}{k}\right) \bar{B}_{q}\left(\frac{\alpha h}{k}\right),
$$

which is called a higher-order Dedekind sum ([1],[8],[9]). For $u, v \in \mathbf{R}$, the sum (5) is also generalized as

$$
\begin{aligned}
& s_{p, q}(h, k: u, v) \\
& =\sum_{\alpha \bmod k} \bar{B}_{p}\left(\frac{\alpha+u}{k}\right) \bar{B}_{q}\left(\frac{(\alpha+u) h}{k}+v\right),
\end{aligned}
$$

which is often called a Dedekind-Rademacher sum and generalizations of (2) are studied in [11], [12] and [23].

In [15], making use of the Fourie expansion of $\bar{B}_{p}(x)$, Halbritter expressed the sum (6) as a series representation and obtained generalizations of (2) which connects the sum (6) and transformed ones of (6) by a unimodular matrix. 
Recently, by a combinatorial-geometric method, which is deeply connected with the theory of lattice points in polytopes ([3], [7]), Beck gave new proofs of (2) and some of its generalizations ([5], [6], [21]). In [19] and [20], motivated by the method, the author studied generalizations of (3) and obtained results including many preceding ones shown in [16], [22], [1], [24],[4], [17] and [18].

In the present paper, in addition to the combinatorial-geometric method, we also make use of the Fourie expansion of $\bar{B}_{p}(x)$ as in [15] and study generalizations of (3) for multiple Dedekind sums attached to Dirichlet characters. The main result is contained in that of [20] as an important example and the significance of this paper can be regarded as giving a new proof of it.

Let us give a description of each section.

In Section 2, we define multiple Dedekind sums by making use of certain generalized Bernoulli functions attached to Dirichlet characters and state the main result. In Section 3, making use of the Fourie expansions of Bernoulli functions, we deduce a series representation of the multiple Dedekind sum. In Section 4, we prove the main result for the special case $s_{p, q}(h, k)$ in order to provide a good overview. In Section 5, we give a complete proof for the general case.

\section{Definitions and the Main Result}

As in the introduction, let $B_{p}(X)$ be the $p$ th Bernoulli polynomial defined by

$$
\frac{t e^{t X}}{e^{t}-1}=\sum_{p=0}^{\infty} B_{p}(X) \frac{t^{p}}{p !} .
$$

and $\bar{B}_{p}(x)$ the $p$ th Bernoulli function defined by (4).

Let $\chi$ be a Dirichlet characters (not necessarily primitive) defined modulo $\mathcal{N}_{\chi}$. The usual $p$ th Bernoulli polynomial $B_{p, \chi}(X)$ attached to $\chi$ is defined by

$$
\sum_{a=0}^{\mathcal{N}_{\chi}-1} \frac{\chi(a) t e^{(X+a) t}}{e^{\mathcal{N}_{\chi} t}-1}=\sum_{p=0}^{\infty} B_{p, \chi}(X) \frac{t^{p}}{p !},
$$

which is equivalent to

$$
B_{p, \chi}\left(\mathcal{N}_{\chi} X\right)=\mathcal{N}_{\chi}^{p-1} \sum_{a \bmod \mathcal{N}_{\chi}} \chi(a) B_{p}\left(X+\frac{a}{\mathcal{N}_{\chi}}\right) \text {. }
$$

On the basis of this equation, we define a function

$$
\beta_{p, \chi}(x)=\sum_{a \bmod \mathcal{N}_{\chi}} \chi(a) \bar{B}_{p}\left(x+\frac{a}{\mathcal{N}_{\chi}}\right) .
$$

For $k=\left(k_{1}, \cdots, k_{r}\right) \in \mathbf{N}^{r} \quad$ we denote by $R_{m}(k) \subset \mathbf{Z}^{r}$ an arbitrarily fixed complete set of representatives of the residue class group $\left(\mathbf{Z} / k_{1} \mathbf{Z}\right) \times \cdots \times\left(\mathbf{Z} / k_{r} \mathbf{Z}\right)$. If $\alpha=\left(\alpha_{1}, \cdots, \alpha_{r}\right)$ $\in \mathbf{Z}^{r}$, we put

$$
\frac{\alpha}{\boldsymbol{k}}=\left(\frac{\alpha_{1}}{k_{1}} \cdots \frac{\alpha_{r}}{k_{r}}\right) .
$$

Let $r, s \in \mathbf{N}$ and let $P=\left(p_{1}, \cdots, p_{r}\right) \in \mathbf{Z}_{\geq 0}^{r}$ and $Q=\left(q_{1}, \cdots, q_{s}\right) \in \mathbf{Z}_{\geq 0}^{s}$. Let $\Phi=\left(\phi_{1}, \cdots, \phi_{r}\right)$ and $\Psi=\left(\psi_{1}, \cdots, \psi_{s}\right)$ be tuples of Dirichlet characters. For $\quad x=\left(x_{1}, \cdots, x_{r}\right) \in \mathbf{R}^{r}$ and $y=\left(y_{1}, \cdots, y_{s}\right)$ $\in \mathbf{R}^{s}$, we put

$$
\begin{gathered}
F(\boldsymbol{x}, \boldsymbol{y}: P, Q, \Phi, \Psi)= \\
\left(\prod_{i=1}^{r} \beta_{p_{i}, \phi_{i}}\left(x_{i}\right)\right) \prod_{j=1}^{s} \beta_{q_{j}, \psi_{j}}\left(y_{j}\right) .
\end{gathered}
$$

Let $H=\left(h_{i j}\right)_{\substack{1 \leq i \leq r \\ 1 \leq j \leq s}}$ be an $r \times s$ matrix with $h_{i j} \in \mathbf{Z}$ for all $1 \leq i \leq r$ and $1 \leq j \leq s$. Note that if $\boldsymbol{\alpha}=\left(\alpha_{1}, \cdots, \alpha_{r}\right) \in \mathbf{Z}^{r}$, then

$$
\begin{aligned}
& F\left(\frac{\boldsymbol{\alpha}}{\boldsymbol{k}}, \frac{\boldsymbol{\alpha}}{\boldsymbol{k}} H: P, Q, \Phi, \Psi\right)=\left(\prod_{i=1}^{r} \beta_{p_{i}, \phi_{i}}\left(\frac{\alpha_{i}}{k_{i}}\right)\right) \\
& \times \prod_{j=1}^{s} \beta_{q_{j}, \psi_{j}}\left(\frac{\alpha_{1} h_{1 j}}{k_{1}}+\cdots+\frac{\alpha_{r} h_{r j}}{k_{r}}\right) .
\end{aligned}
$$

We define a multiple Dedekind sum as

$$
\begin{aligned}
& S(H, \boldsymbol{k}: P, Q, \Phi, \Psi) \\
& =\sum_{\alpha \in R_{m}(k)} F\left(\frac{\boldsymbol{\alpha}}{\boldsymbol{k}}, \frac{\boldsymbol{\alpha}}{\boldsymbol{k}} H: P, Q, \Phi, \Psi\right) .
\end{aligned}
$$

The sum (7) is an important example of a generalized Dedekind type sum defined as (2.3) in [20] 
and includes (1), (5) and some of the generalized Dedekind sums appearing in [4], [24], [18], [19] etc. Note that if we define a twisted $p$ th Bernoulli function $\tilde{B}_{p, \chi}(x)$ for $x \in \mathbf{Q}$ with denominators relatively prime to $\mathcal{N}_{\chi}$ by

$$
\tilde{B}_{p, \chi}(x)=\mathcal{N}_{\chi}^{p-1} \sum_{a \bmod \mathcal{N}_{\chi}} \chi(x+a) \bar{B}_{p}\left(\frac{x+a}{\mathcal{N}_{\chi}}\right)
$$

as in Section 2 of [20], then we have $\tilde{B}_{p, \chi}\left(\mathcal{N}_{\chi} x\right)=\mathcal{N}_{\chi}^{p-1} \beta_{p, \chi}(x)$. If we put

$$
F(x, y)=\left(\prod_{i=1}^{r} \tilde{B}_{p_{i}, \phi_{i}}\left(x_{i}\right)\right) \prod_{j=1}^{s} \tilde{B}_{q_{j}, \psi_{j}}\left(y_{j}\right),
$$

then the relation between (7) and $S(F: H, k)$ in [20] is verified as follows:

Suppose $\operatorname{gcd}\left\{\mathcal{N}_{\phi_{i}} \mathcal{N}_{\psi_{j}}, k_{1} \cdots k_{r}\right\}=1$ for all $1 \leq i \leq r$ and $1 \leq j \leq s$ and let $H^{\prime}=\left(h^{\prime}{ }_{i j}\right)_{\substack{1 \leq i \leq r \\ 1 \leq j \leq s}}$ be an $r \times s$ matrix with $h_{i j}^{\prime} \in \mathbf{Z}$ such that $\mathcal{N}_{\phi_{i}} h^{\prime}{ }_{i j} \equiv \mathcal{N}_{\psi_{j}} h_{i j}\left(\bmod k_{i}\right)$ holds for all $1 \leq i \leq r$ and $1 \leq j \leq s$. Then it is easy to see that

$$
\begin{aligned}
& S(H, k: P, Q, \Phi, \Psi) \\
& =\left(\prod_{i=1}^{r} \mathcal{N}_{\phi_{i}}^{1-p_{i}}\right)\left(\prod_{j=1}^{s} \mathcal{N}_{\psi_{j}}^{1-q_{j}}\right) S\left(F: H^{\prime}, k\right) .
\end{aligned}
$$

Now for any Dirichlet character $\chi$ and any $l, N \in \mathbf{N}$, we put

$$
\sigma_{l, \chi}(N)=\sum_{\delta \mid N} \chi(\delta) \delta^{l} .
$$

For any $d \in \mathbf{N}$, let $\mathcal{I}_{d}(r, s)$ be a set of $r \times s$ matrices such as

$$
\mathcal{I}_{d}(r, s)=\left\{B=\left(b_{i j}\right)_{\substack{1 \leq i \leq r \\ 1 \leq j \leq s}} \mid b_{i j} \in \mathbf{Z}, 0 \leq b_{i j} \leq d-1\right.
$$

for all $1 \leq i \leq r$ and $1 \leq j \leq s\}$.

For $k=\left(k_{1}, \cdots, k_{r}\right) \in \mathbf{N}^{r}$ and $B \in \mathcal{I}_{d}(r, s)$, we put

$$
k \circ B=\left(\begin{array}{lll}
k_{1} & & 0 \\
& \ddots & \\
0 & & k_{r}
\end{array}\right) B
$$

which is a matrix obtained from $B$ by multiplying the $i$ th row by $k_{i}$ for each $1 \leq i \leq r$. Put

$$
\begin{aligned}
& s(P)=\sum_{i=1}^{r} p_{i}, s(Q)=\sum_{j=1}^{s} q_{j}, \\
& s(P, Q)=s(P)+s(Q), \\
& \phi=\prod_{i=1}^{r} \phi_{i} \text { and } \psi=\prod_{j=1}^{s} \psi_{j} .
\end{aligned}
$$

Then the main result is the following.

Theorem 2.1 Let $N \in \mathbf{N}$ with $\operatorname{gcd}\left\{N, \mathcal{N}_{\phi_{i}}\right\}=\operatorname{gcd}\left\{N, \mathcal{N}_{\psi_{j}}\right\}=1$ for all $1 \leq i \leq r$ and $1 \leq j \leq s$. Then we have

$$
\begin{aligned}
& N^{s(P)-r} \phi(N) \sum_{\substack{a d=N \\
d>0}} \sum_{B \in \mathcal{I}_{d}(r, s)} d^{s(Q)-s r} \psi(d) \\
& \times S(a H+k \circ B, d k: P, Q, \Phi, \Psi) \\
& =\sigma_{s(P, Q)-r, \phi \psi}(N) S(H, k: P, Q, \Phi, \Psi) .
\end{aligned}
$$

\section{Series Representations of Dedekind Sums}

For $p \in \mathbf{N}$, the Fourie expansion of $\bar{B}_{p}(x)$ is expressed as

$$
\bar{B}_{p}(x)=-\frac{p !}{(2 \pi \sqrt{-1})^{p}} \lim _{N \rightarrow \infty} \sum_{\substack{n=-N \\ n \neq 0}}^{N} \frac{\exp (2 \pi \sqrt{-1} n x)}{n^{p}} .
$$

In order to include the case of $p=0$, we put

$c_{p}(n)=\left\{\begin{array}{cl}n^{-p} & \text { if } p \geq 1 \text { and } n \in \mathbf{Z} \text { with } n \neq 0 \\ -1 & \text { if } p=0 \text { and } n=0 \\ 0 & \text { otherwise. }\end{array}\right.$

We put further

$$
e(x)=\exp (2 \pi \sqrt{-1} x) .
$$

Then for all $p \geq 0$, we have

$$
\bar{B}_{p}(x)=-\frac{p !}{(2 \pi \sqrt{-1})^{p}} \lim _{N \rightarrow \infty} \sum_{n=-N}^{N} c_{p}(n) e(n x) .
$$

In addition, for any fixed $N_{1}, N_{2} \in \mathbf{Z}$, we also 
have

$$
\bar{B}_{p}(x)=-\frac{p !}{(2 \pi \sqrt{-1})^{p}} \lim _{N \rightarrow \infty} \sum_{n=-N+N_{1}}^{N+N_{2}} c_{p}(n) e(n x) .
$$

As in Section 2, let $\chi$ be a Dirichlet character defined modulo $N_{\chi}$. Put $\zeta_{\chi}=e\left(1 / \mathcal{N}_{\chi}\right)$ and

$$
\tau\left(\chi, \zeta_{\chi}^{n}\right)=\sum_{b \bmod \mathcal{N}_{\chi}} \chi(b) \zeta_{\chi}^{n b}
$$

for $n \in \mathbf{Z}$ and define

$$
c_{p, \chi}(n)=\tau\left(\chi, \zeta_{\chi}^{n}\right) c_{p}(n)
$$

Then, direct calculation shows that

$$
\beta_{p, \chi}(x)=-\frac{p !}{(2 \pi \sqrt{-1})^{p}} \lim _{N \rightarrow \infty} \sum_{n=-N+N_{1}}^{N+N_{2}} c_{p, \chi}(n) e(n x)
$$

for any fixed $N_{1}, N_{2} \in \mathbf{Z}$. If $\chi$ is primitive, we have $\quad c_{p, \chi}(n)=\chi^{-1}(n) \tau\left(\chi, \zeta_{\chi}\right) c_{p}(n) \quad$ for all $n \in \mathbf{Z}$ and so,

$$
\begin{aligned}
& \beta_{p, \chi}(x)=-\frac{p !}{(2 \pi \sqrt{-1})^{p}} \tau\left(\chi, \zeta_{\chi}\right) \\
& \times \lim _{N \rightarrow \infty} \sum_{n=-N+N_{1}}^{N+N_{2}} \chi^{-1}(n) c_{p}(n) e(n x) . \\
& \text { For } \quad P=\left(p_{1}, \cdots, p_{r}\right) \in \mathbf{Z}_{\geq 0}^{r} \quad \text { and } \\
& Q=\left(q_{1}, \cdots, q_{s}\right) \in \mathbf{Z}_{\geq 0}^{s}, \text { we put } \\
& A(P, Q)=(-1)^{r+s}\left(\prod_{i=1}^{r} p ! \prod_{j=1}^{s} q !\right)^{-1}(2 \pi \sqrt{-1})^{s(P, Q)}
\end{aligned}
$$

and

$$
\begin{aligned}
& T(H, \boldsymbol{k}: P, Q, \Phi, \Psi)= \\
& A(P, Q) S(H, \boldsymbol{k}: P, Q, \Phi, \Psi) .
\end{aligned}
$$

Especially, we put

$$
t_{p, q}(h, k)=(p ! q !)^{-1}(2 \pi \sqrt{-1})^{p+q} s_{p, q}(h, k)
$$

for $k \in \mathbf{N}, h \in \mathbf{Z}$ and $p, q \in \mathbf{Z}_{\geq 0}$. Then we have the following.

Lemma 3.1 We have

$$
\begin{aligned}
& t_{p, q}(h, k) \\
& =k \lim _{M \rightarrow \infty} \sum_{m=-M}^{M} c_{q}(m)\left(\lim _{L \rightarrow \infty} \sum_{l=-L}^{L} c_{p}(l k-m h)\right) .
\end{aligned}
$$

and

$$
\begin{aligned}
& T(H, \boldsymbol{k}: P, Q, \Phi, \Psi) \\
& =k_{1} \cdots k_{r} \lim _{M \rightarrow \infty} \sum_{\substack{-M \leq m_{j} \leq M \\
(1 \leq j \leq s)}}\left(\prod_{j=1}^{s} c_{q_{j}, \psi_{j}}\left(m_{j}\right)\right) \\
& \times\left(\lim _{L \rightarrow \infty} \sum_{\substack{-L \leq l_{i} \leq L \\
(1 \leq i \leq r)}} \prod_{i=1}^{r} c_{p_{i}, \phi_{i}}\left(l_{i} k_{i}-m_{1} h_{i 1}-\cdots-m_{s} h_{i s}\right)\right) .
\end{aligned}
$$

Proof: By (8) and the definition of $t_{p, q}(h, k)$, we see that

$$
\begin{aligned}
t_{p, q}(h, k) & =\lim _{M \rightarrow \infty} \sum_{m=-M}^{M} c_{q}(m) \\
\times & \left(\lim _{N \rightarrow \infty} \sum_{n=-N}^{N} c_{p}(n) \sum_{\alpha \bmod k} e\left(\frac{(n+m h) \alpha}{k}\right)\right) .
\end{aligned}
$$

Note that

$$
\sum_{\alpha \bmod k} e\left(\frac{(n+m h) \alpha}{k}\right)= \begin{cases}k & \text { if } n \equiv-m h \bmod k \\ 0 & \text { otherwise }\end{cases}
$$

Hence, taking (9) into account, we obtain (10). In the same way, we also obtain (11).

\section{Remark.}

(1) The formula (10) is contained in equation (13) of [15], which is the series representation of (6).

(2) In the summations of the right hand side of (10), we can replace $m$ and $l$ by $-m$ and $-l$, respectively and see that

$$
\begin{aligned}
& t_{p, q}(h, k)=k \lim _{M \rightarrow \infty} \sum_{m=-M}^{M} c_{q}(-m) \\
& \times\left(\lim _{L \rightarrow \infty} \sum_{l=-L}^{L} c_{p}(-l k+m h)\right) \\
& =(-1)^{p+q} t_{p, q}(h, k) .
\end{aligned}
$$

In particular, if $p \not \equiv q \bmod 2$, we have $t_{p, q}(h, k)=0$. In the same way, we have 


$$
\begin{gathered}
T(H, \boldsymbol{k}: P, Q, \Phi, \Psi)= \\
(-1)^{s(P, Q)} S(H, \boldsymbol{k}: P, Q, \Phi, \Psi) .
\end{gathered}
$$

and if $s(P) \not \equiv s(Q) \bmod 2$, we have

$$
T(H, \boldsymbol{k}: P, Q, \Phi, \Psi)=0 .
$$

\section{Proof of Theorem 2.1 in a Special Case}

In this section, we prove theorem 2.1 in a special case of $r=s=1$ and $\phi=\psi=1$. In this case, theorem 2.1 reduces to the following formula:

$$
\begin{aligned}
& N^{p-1} \sum_{\substack{a d=N \\
d>0}} d^{q-1} \sum_{b=0}^{d-1} t_{p, q}(a h+k b, d k) \\
& =\sigma_{p+q-1}(N) t_{p, q}(h, k) .
\end{aligned}
$$

In [19] and [20], we introduced an additive subgroup of $\mathbf{Z}^{2}$ such as

$$
\mathcal{A}(a, d: b)=(a,-b) \mathbf{Z}+(0, d) \mathbf{Z}
$$

for $a, d \in \mathbf{N}$ and $b \in \mathbf{Z}$ and by considering conditions for $(m, l) \in \mathbf{Z}^{2}$ to be in $\mathcal{A}(a, d: b)$, we deduced the following (lemma 3.3 of [20]).

Lemma 4.1 Let $R$ be a ring extension of $\mathbf{Q}$ and let $\mathcal{F}$ be a map from $\mathbf{Z}^{2}$ to $R$. Then for any finite subset $F$ of $\mathbf{Z}^{2}$ and for any $N \in \mathbf{N}$, we have

$$
\begin{aligned}
& \sum_{\substack{a d=N \\
d>0}} \sum_{b=0}^{d-1} \sum_{(m, l) \in \mathcal{A}(a, d: b) \cap F} \mathcal{F}(m, l) \\
& =\sum_{\delta \mid N} \delta \sum_{(m, l) \in \delta \mathbf{Z}^{2} \cap F} \mathcal{F}(m, l) .
\end{aligned}
$$

Let us deduce (12) from (10) and lemma 4.1. Put

$$
\mathcal{F}(m, l: h, k)=c_{q}(m) c_{p}(l k-m h) .
$$

Then

$$
t_{p, q}(h, k)=k \lim _{M \rightarrow \infty}\left(\lim _{L \rightarrow \infty} \sum_{m=-M}^{M} \sum_{l=-L}^{L} \mathcal{F}(m, l: h, k)\right) \text {. }
$$

Note that we have

$$
\mathcal{F}(\delta m, \delta l: h, k)=\delta^{-p-q} \mathcal{F}(m, l: h, k)
$$

for any $\delta \in \mathbf{N}$ and that for any fixed $L_{1}$ and $L_{1}^{\prime} \in \mathbf{Z}$, we also have

$$
\begin{aligned}
& t_{p, q}(h, k) \\
& =k \lim _{M \rightarrow \infty}\left(\lim _{L \rightarrow \infty} \sum_{m=-M}^{M} \sum_{l=-L+L_{1}}^{L+L_{1}} \mathcal{F}(m, l: h, k)\right) .
\end{aligned}
$$

For $N, M, L \in \mathbf{N}$, we put

$$
\begin{aligned}
& \mathcal{R}_{N}(M, L) \\
& =\left\{(m, l) \in \mathbf{Z}^{2} \mid-M N \leq m \leq M N,\right. \\
& -L N \leq l \leq L N\} .
\end{aligned}
$$

If $(m, l) \in \mathcal{A}(a, d: b)$, we can express

$$
(m, l)=(a,-b) \mu+(0, d) v=(a \mu,-b \mu+d v)
$$

with $\mu, v \in \mathbf{Z}$. Put $N=a d$. Then

$$
\begin{aligned}
\mathcal{F}(m, l: h, k) & =c_{q}(a \mu) c_{p}((-b \mu+d v) k-a \mu h) \\
& =a^{-q} c_{q}(\mu) c_{p}(v d k-(a h+k b) \mu) \\
& =\frac{d^{q}}{N^{q}} \mathcal{F}(\mu, v: a h+k b, d k) .
\end{aligned}
$$

Taking $F=\mathcal{R}_{N}(M, L)$, we see that

$$
\begin{gathered}
\sum_{\substack{a d=N \\
d>0}} \sum_{b=0}^{d-1} \sum_{(m, l) \in \mathcal{A}(a, d: b) \cap F} \mathcal{F}(m, l: h, k) \\
=\frac{1}{N^{q}} \sum_{\substack{a d=N \\
d>0}} d^{q} \sum_{b=0}^{d-1} \sum_{\mu=-M d}^{M d}
\end{gathered}
$$

$$
\times \sum_{\substack{v \in \mathbf{Z} \text { with } \\-L N \leq-b \mu+d v \leq L N}} \mathcal{F}(\mu, v: a h+k b, d k)
$$

$$
\begin{gathered}
=\frac{1}{N^{q}} \sum_{\substack{a d=N \\
d>0}} d^{q} \sum_{b=0}^{d-1} \sum_{\mu=-M d}^{M d} \\
\times \sum_{v=-L a-[-b \mu / d]}^{L a+[b \mu / d]} \mathcal{F}(\mu, v: a h+k b, d k) .
\end{gathered}
$$


We also see from (13) that

$$
\begin{aligned}
& \sum_{\delta \mid N} \delta \sum_{(m, l) \in \delta \mathbf{Z}^{2} \cap F} \mathcal{F}(m, l: h, k) \\
& =\sum_{\delta \mid N} \delta \sum_{m=-M N / \delta}^{M N / \delta} \sum_{l=-L N / \delta}^{L N / \delta} \mathcal{F}(\delta m, \delta l: h, k) \\
& =\sum_{\delta \mid N} \delta^{1-p-q} \sum_{m=-M N / \delta}^{M N / \delta} \sum_{l=-L N / \delta}^{L N / \delta} \mathcal{F}(m, l: h, k) .
\end{aligned}
$$

Note that if $\delta$ ranges the divisors of $N$, then so does $N / \delta$. It follows that

$$
\begin{aligned}
& \sum_{\delta \mid N} \delta \sum_{(m, l) \in \delta \mathbf{Z}^{2} \cap F} \mathcal{F}(m, l: h, k) \\
& =N^{1-p-q} \sum_{\delta \mid N} \delta^{p+q-1} \sum_{m=-M \delta}^{M \delta} \sum_{l=-L \delta}^{L \delta} \mathcal{F}(m, l: h, k) .
\end{aligned}
$$

Taking limits for (15) and (16), we see from (14) that

$$
\begin{aligned}
& \lim _{M \rightarrow \infty}\left(\lim _{L \rightarrow \infty} \sum_{\substack{a d=N \\
d>0}} \sum_{b=0}^{d-1} \sum_{(m, l) \in \mathcal{A}(a, d: b) \cap F} \mathcal{F}(m, l: h, k)\right) \\
& =\frac{1}{k N^{q}} \sum_{\substack{a d=N \\
d>0}} d^{q-1} \sum_{b=0}^{d-1} t_{p, q}(a h+k b, d k)
\end{aligned}
$$

and

$$
\begin{aligned}
& \lim _{M \rightarrow \infty}\left(\lim _{L \rightarrow \infty} \sum_{\delta \mid N} \delta \sum_{(m, l) \in \delta \mathbf{Z}^{2} \cap F} \mathcal{F}(m, l: h, k)\right) \\
& =\frac{N^{1-p-q}}{k} \sigma_{p+q-1}(N) t_{p, q}(h, k) .
\end{aligned}
$$

By lemma 4.1, (17) equals (18). Hence, we obtain (12).

\section{Proof of Theorem 2.1 in the General Case}

The method in the previous section is naturally generalized to the general case.

For $a, d \in \mathbf{N}$ and $b \in \mathbf{Z}$, the additive group $\mathcal{A}(a, d: b)$ in the previous section is also expressed as

$$
A(a, d: b)=\left\{(\mu v)\left(\begin{array}{cc}
a & -b \\
0 & d
\end{array}\right) \mid \mu, v \in \mathbf{Z}\right\} .
$$

Let $B$ be an $s \times r$ matrix with components in
$\mathbf{Z}$. We define an additive subgroup of $\mathbf{Z}^{s} \times \mathbf{Z}^{r}$ as

$$
\begin{aligned}
& \mathcal{A}(a, d: B) \\
& =\left\{\left(\begin{array}{ll}
\boldsymbol{\mu} & \boldsymbol{v}
\end{array}\right)\left(\begin{array}{cc}
a E_{s} & -B \\
O & d E_{r}
\end{array}\right) \mid \boldsymbol{\mu} \in \mathbf{Z}^{s}, \boldsymbol{v} \in \mathbf{Z}^{r}\right\},
\end{aligned}
$$

where $E_{s}$ and $E_{r}$ are unit matrices of degrees $s$ and $r$, respectively. As shown in [20], lemma 4.1 is generalized as the following (lemma 4.3 of [20]).

Lemma 5.1 Let $R$ be a ring extension of $\mathbf{Q}$. Let $\mathcal{F}$ be a map from $\mathbf{Z}^{s} \times \mathbf{Z}^{r}$ to $R$. Then for any finite subset $F$ of $\mathbf{Z}^{s} \times \mathbf{Z}^{r}$ and for any $N \in \mathbf{N}$, we have

$$
\begin{aligned}
& \sum_{\substack{a d=N \\
d>0}} \sum_{B \in I_{d}(s, r)} \sum_{(\boldsymbol{m} \boldsymbol{l}) \in A(a, d: B) \cap F} a^{(s-1) r} \mathcal{F}(\boldsymbol{m} \boldsymbol{l}) \\
& =N^{(s-1) r} \sum_{\delta \mid N} \delta^{r} \sum_{(\boldsymbol{m} \boldsymbol{l}) \in\left(\delta \mathbf{Z}^{s} \times \delta \mathbf{Z}^{r}\right) \cap F} \mathcal{F}(\boldsymbol{m} \boldsymbol{l}) .
\end{aligned}
$$

Let us deduce theorem 2.1 from (11) and lemma 5.1. For $\boldsymbol{m}=\left(m_{1}, \cdots, m_{s}\right) \in \mathbf{Z}^{s}, \boldsymbol{l}=\left(l_{1}, \cdots, l_{r}\right) \in \mathbf{Z}^{r}$ and $\boldsymbol{k}=\left(k_{1}, \cdots, k_{r}\right) \in \mathbf{N}^{r}$, we put $F(\boldsymbol{m}, \boldsymbol{l}: H, \boldsymbol{k})=\left(\prod_{j=1}^{s} c_{q_{j}, \psi_{j}}\left(m_{j}\right)\right)$
$\times \prod_{i=1}^{r} c_{p_{i}, \phi_{i}}\left(l_{i} k_{i}-m_{1} h_{i 1}-\cdots-m_{s} h_{i s}\right)$.

Then

$$
\begin{aligned}
& T(H, \boldsymbol{k}: P, Q, \Phi, \Psi)=k_{1} \cdots k_{r} \\
& \times \lim _{M \rightarrow \infty}\left(\lim _{L \rightarrow \infty} \sum_{\substack{-M \leq m_{j} \leq M \\
(1 \leq j \leq s)}} \sum_{\substack{L \leq l_{i} \leq L \\
(1 \leq i \leq r)}} \mathcal{F}(\boldsymbol{m}, \boldsymbol{l}: H, \boldsymbol{k})\right) .
\end{aligned}
$$

Note that we have

$$
\begin{aligned}
& \mathcal{F}(\delta \boldsymbol{m}, \delta \boldsymbol{l}: H, \boldsymbol{k})= \\
& \left(\delta^{s(P, Q)} \phi \psi(\delta)\right)^{-1} \mathcal{F}(\boldsymbol{m}, \boldsymbol{l}: H, \boldsymbol{k})
\end{aligned}
$$

for any $\delta \in \mathbf{N}$ and that for any fixed $L_{1}, L_{1}^{\prime}, \cdots L_{r}, L_{r}^{\prime} \in \mathbf{Z}$, we also have

$$
\begin{aligned}
& T(H, \boldsymbol{k}: P, Q, \Phi, \Psi)=k_{1} \cdots k_{r} \\
\times & \lim _{M \rightarrow \infty}\left(\lim _{L \rightarrow \infty} \sum_{\substack{-M \leq m_{j} \leq M \\
(1 \leq j \leq s)}} \mathcal{F}(\boldsymbol{m}, \boldsymbol{l}: H, \boldsymbol{k})\right) . \\
& \sum_{\substack{-L+L_{i} \leq l_{i} \leq L+L_{i}^{\prime} \\
(1 \leq i \leq r)}}
\end{aligned}
$$


For $N, M, L \in \mathbf{N}$, we put

$$
\begin{aligned}
& \mathcal{R}_{N}(M, L)=\left\{(\boldsymbol{m}, \boldsymbol{l}) \in \mathbf{Z}^{s} \times \mathbf{Z}^{r} \mid \boldsymbol{m}\right. \\
& =\left(m_{1}, \cdots, m_{s}\right), \boldsymbol{l}=\left(l_{1}, \cdots, l_{r}\right) \\
& \quad \text { with }-M N \leq m_{j} \leq M N,-L N \leq l_{i} \leq L N \\
& \quad \text { for } 1 \leq j \leq s, 1 \leq i \leq r\} .
\end{aligned}
$$

If $B=\left(b_{i j}\right)_{\substack{1 \leq i \leq r \\ 1 \leq j \leq s}}$ is an $r \times s$ matrix with components in $\mathbf{Z}$, then the transposed matrix ${ }^{t} B$ of $B$ is an $s \times r$ matrix and $(\boldsymbol{m} l) \in \mathcal{A}\left(a, d:^{t} B\right)$ is expressed as

$$
(\boldsymbol{m} \boldsymbol{l})=(\boldsymbol{\mu} \boldsymbol{v})\left(\begin{array}{cc}
a E_{s} & -{ }^{t} B \\
O & d E_{r}
\end{array}\right)
$$

with $\boldsymbol{\mu} \in \mathbf{Z}^{s} \quad$ and $\quad \boldsymbol{v} \in \mathbf{Z}^{r}$. If $\boldsymbol{m}=\left(m_{1}, \cdots, m_{s}\right), \boldsymbol{l}=\left(l_{1}, \cdots, l_{r}\right), \boldsymbol{\mu}=\left(\mu_{1}, \cdots, \mu_{s}\right)$ and $\boldsymbol{v}=\left(v_{1}, \cdots, v_{r}\right)$, then

$$
m_{j}=a \mu_{j} \quad \text { and } \quad l_{i}=-\mu_{1} b_{i 1}-\cdots-\mu_{s} b_{i s}+v_{i} d
$$

for $1 \leq j \leq s$ and $1 \leq i \leq r$. Hence, by direct calculations, we see that

$\mathcal{F}(\boldsymbol{m}, \boldsymbol{l}: H, \boldsymbol{k})=$

$\frac{d^{s(Q)} \psi(d)}{N^{s(Q)} \psi(N)} \mathcal{F}(\boldsymbol{\mu}, \boldsymbol{v}: a H+\boldsymbol{k} \circ B, d \boldsymbol{k})$,

where $N=a d$. Taking $F=\mathcal{R}_{N}(M, L)$, we see that

$$
\begin{aligned}
& \sum_{\substack{a d=N \\
d>0}} \sum_{B \in \mathcal{I}_{d}(r, s)} \sum_{(\boldsymbol{m} l) \in A\left(a, d::^{t} B\right) \cap F} a^{(s-1) r} \mathcal{F}(\boldsymbol{m}, \boldsymbol{l}: H, \boldsymbol{k}) \\
= & \frac{1}{N^{s(Q)-(s-1) r} \psi(N)} \sum_{\substack{a d=N \\
d>0}} d^{s(Q)-(s-1) r} \psi(d) \\
& \times \sum_{B \in \mathcal{I}_{d}(r, s)} \sum_{\mu} \sum_{v} F(\boldsymbol{\mu}, \boldsymbol{v}: a H+\boldsymbol{k} \circ B, d \boldsymbol{k}),
\end{aligned}
$$

where in the above summations, $\boldsymbol{\mu}=\left(\mu_{1}, \cdots, \mu_{s}\right)$ ranges in $\mathbf{Z}^{s}$ such that $-M d \leq \mu_{j} \leq M d$ for $1 \leq j \leq s$ and $\boldsymbol{v}=\left(v_{1}, \cdots, v_{r}\right)$ in $\mathbf{Z}^{r}$ such that $-L N \leq-\mu_{1} b_{i 1}-\cdots-\mu_{s} b_{i s}+v_{i} d \leq L N \quad$ for $1 \leq i \leq r$, namely

$$
\begin{aligned}
& -L a-\left[-\left(\mu_{1} b_{i 1}+\cdots+\mu_{s} b_{i s}\right) / d\right] \\
& \leq v_{i} \leq L a+\left[\left(\mu_{1} b_{i 1}+\cdots+\mu_{s} b_{i s}\right) / d\right] .
\end{aligned}
$$

We also see that

$$
\begin{aligned}
& N^{(s-1) r} \sum_{\delta \mid N} \delta^{r} \sum_{(\boldsymbol{m} \boldsymbol{l}) \in\left(\delta \mathbf{Z}^{s} \times \delta \mathbf{Z}^{r}\right) \cap F} \mathcal{F}(\boldsymbol{m}, \boldsymbol{l}: H, \boldsymbol{k}) \\
= & N^{(s-1) r} \sum_{\delta \mid N} \delta^{r} \sum_{(\boldsymbol{m} \boldsymbol{l}) \in\left(\mathbf{Z}^{s} \times \mathbf{Z}^{r}\right) \cap \frac{1}{\delta} F} \mathcal{F}(\delta \boldsymbol{m} \delta \boldsymbol{l}: H, k) \\
= & N^{(s-1) r} \sum_{\delta \mid N} \delta^{r-s(P, Q)}(\phi \psi(\delta))^{-1} \\
\times & \sum_{(\boldsymbol{m} \boldsymbol{l}) \in\left(\mathbf{Z}^{s} \times \mathbf{Z}^{r}\right) \cap \frac{1}{\delta} F} \mathcal{F}(\boldsymbol{m} \boldsymbol{l}: H, \boldsymbol{k})(\mathrm{by}(19)) \\
= & \frac{N^{s r-s(P, Q)}}{\phi} \sum_{\psi(N)} \sum_{\delta \mid N} \delta^{s(P, Q)-r} \phi \psi(\delta) \\
\times & \sum_{(\boldsymbol{m} \boldsymbol{l}) \in\left(\mathbf{Z}^{s} \times \mathbf{Z}^{r}\right) \cap \frac{\delta}{N} F} \mathcal{F}(\boldsymbol{m}, \boldsymbol{l}: H, \boldsymbol{k}) .
\end{aligned}
$$

Taking limits for (21) and (22), we see from (20) that

$$
\begin{aligned}
& \lim _{M \rightarrow \infty}\left(\lim _{L \rightarrow \infty} \sum_{\substack{a d=N \\
d>0}} \sum_{B \in \mathcal{I}_{d}(r, s)}\right. \\
& \left.\sum_{(\boldsymbol{m} \boldsymbol{l}) \in A\left(a, d^{t}:^{\prime} B\right) \cap F} a^{(s-1) r} \mathcal{F}(\boldsymbol{m} \boldsymbol{l}: H, \boldsymbol{k})\right) \\
& =\frac{1}{N^{s(Q)-(s-1) r} \psi(N)} \sum_{\substack{a d=N \\
d>0}} \frac{d^{s(Q)-(s-1) r} \psi(d)}{d^{r} k_{1} \cdots k_{r}} \\
& \quad \times \sum_{B \in \mathcal{I}_{d}(r, s)} T(a H+\boldsymbol{k} \circ B, d \boldsymbol{k}: P, Q, \Phi, \Psi)
\end{aligned}
$$

and

$$
\begin{aligned}
& \lim _{M \rightarrow \infty}\left(\lim _{L \rightarrow \infty} N^{(s-1) r} \sum_{\delta \mid N} \delta^{r}\right. \\
& \left.\quad \times \sum_{(\boldsymbol{m} l) \in\left(\delta \mathbf{Z}^{s} \times \delta \mathbf{Z}^{r}\right) \cap F} \mathcal{F}(\boldsymbol{m} \mathbf{l}: H, \boldsymbol{k})\right) \\
& =\frac{N^{s r-s(P, Q)}}{\phi \psi(N) k_{1} \cdots k_{r}} \sigma_{s(P, Q)-r, \phi \psi}(N) \\
& \times T(H, \boldsymbol{k}: P, Q, \Phi, \Psi) .
\end{aligned}
$$

By lemma 5.1, (23) equals (24). Hence theorem 2.1 follows by the definition of $T(H, \boldsymbol{k}: P, Q, \Phi, \Psi)$. 


\section{References}

[1] T. M. Apostol, Generalized Dedekind sums and transformation formulae of certain Lambert series, Duke Math. J. 17 (1950), 147-157.

[2] T. M. Apostol, T. H. Vu, Identities for sums of Dedekind type, J. Number Theory 14 (1982), 391-396.

[3] A. Barvinok, J. E. Pommersheim, An algorithmic theory of lattice points in polyhedra. New perspectives in algebraic combinatorics (Berkeley, CA, 1996-97), 91-147.

[4] M. Beck, Dedekind cotangent sums, Acta Arith. 109 (2003), 109-130.

[5] M. Beck, Geometric proofs of polynomial reciprocity laws of Carlitz, Berndt and Dieter, Diophantine analysis and related field 2006, Sem. Math. Sci. Keio Univ. 35 (2006), 11-18.

[6] M. Beck, C. Haase, A. R. Matthews, Dedekind-Carlitz polynomials as lattice-point enumerators in rational polyhedra, Math. Ann. 341 (2008), 945-961.

[7] M. Beck, S. Robins, Computing the continuous discretely: Integerpoint enumeration in polyhedra, Undergraduate Texts in Mathematics, Springer, New York, 2007.

[8] L. Carlitz, Some theorems on generalized Dedekind sums, Pacific J. of Math. 3 (1953), 513-522.

[9] L. Carlitz, Dedekind sums and Lambert series, Proc. Amer. Math. Soc. 5 (1954), 580-584.

[10] L. Carlitz, A note on generalized Dedekind sums, Duke. Math. J. 21 (1954), 399-403.

[11] L. Carlitz, Generalized Dedekind sums, Math. Zeit. 85 (1964), 83-90.

[12] L. Carlitz, A theorem on generalized Dedekind sums,
Acta Arith. 11 (1965), 253-260.

[13] L. Carlitz, Many-term relations for multiple Dedekind sums, Indian J. Math. 20 (1978), 77-89.

[14] R. Dedekind, Erläuterungen zu zwei Fragmenten von Riemann, Gesammelte mathematische Werke, Bd. I. S, 159-173.

[15] U. Halbritter, Some new reciprocity formulas for generalized Dedekind sums, Results in Math. 8 (1985), 21-46.

[16] M. I. Knopp, Hecke operators and an identity for the Dedekind sums, J. Number Theory 12 (1980), 2-9.

[17] K. Kozuka, Dedekind type sums attached to Dirichlet characters, Kyusyu J. Math. 58 (2004), 1-24.

[18] K. Kozuka, Some identities for multiple Dedekind sums attached to Dirichlet characters, Acta Arith. 146 (2011), 103-114.

[19] K. Kozuka, Combinatorial-geometric viewpoint of Knopp's formula for Dedekind sums, Functiones et Approximatio 46 (2012), 79-89.

[20] K. Kozuka, Knopp-type identities for generalized Dedekind-type sums, Kyusyu J. Math. 68 (2014), 405-419.

[21] A. R. Matthews, A Geometric Approach to Carlitz-Dedekind sums, San Francisco State University 2007.

[22] L. A. Parson, K. H. Rosen, Hecke operators and Lambert series, Math. Scand. 49 (1981), 5-14.

[23] H. Rademacher, Some remarks on certain generalized Dedekind sums, Acta Arith. 9 (1964), 97-105.

[24] Z. Zheng, The Petersson-Knopp indentity for the homogeneous Dedekind sums. J. Number Theory 57 (1996), 223-230. 